\title{
Role of Sphincterotomy in Small Pupil in SICS
}

Manisha Rathi, MS ${ }^{1 *}$, Dr. Ritesh Verma, $\mathrm{MS}^{2}$, Dr. Dixit Soni, $\mathrm{MBBS}^{2}$, Dr. Inder Mohan Rustagi, MS ${ }^{3}$, Sumit Sachdeva, MS ${ }^{1}$, Dr. Jitender Phogat, MS ${ }^{1}$

${ }^{1}$ Professor, Regional Institute of Ophthalmology, Pt BD Sharma PGIMS, Rohtak, Haryana, India

${ }^{2}$ Regional Institute of Ophthalmology, Pt BD Sharma PGIMS, Rohtak, Haryana, India

${ }^{3}$ Triveni Hospitals PVT. LTD. Gurugram, Haryana, India

DOI: $10.36347 /$ sjams.2021.v09i01.013

| Received: 27.12.2020 | Accepted: 12.01.2021 | Published: 14.01.2021

*Corresponding author: Manisha Rathi

Abstract

Original Research Article

Dealing with a rigid non dilating pupil in a small incision cataract surgery is challenging, even more so when dealing with a large and a hard nucleus. There is an increased risk of complication in eyes with small pupil $(<6 \mathrm{~mm})$ during cataract surgery. Loose zonules and hard nuclei maybe missed in small pupils, leading to complications. Aspiration of cortical matter and capsulorrhexis behind the iris in spaces that are not visible to the surgeon can cause an increased risk of complications. In India, where the majority of the people visiting a government tertiary care hospital like ours present with white/hard cataract belong to a lower socio-economic strata, there is a need of an effective and inexpensive method that provides adequate pupillary dilation during cataract surgery. There are various methods described to make the pupil wider during surgery, like iris sutures, Healon 5, iris hooks, mechanical stretching, and ring expanders, which require additional instruments and cost. This is an added burden for the majority of the patients who are of a low socioeconomic status and present late with white/hard cataracts. We decided to study the efficacy and safety of sphincterotomy in white/hard cataracts who underwent SICS in our unit. This study on 20 eyes showed that sphincterotomy is a safe, easy to perform method without any additional cost to obtain an adequate pupil size in small pupils and has excellent postoperative results.

Keywords: Sphincterotomy; rigid pupil; hard cataract, small pupil, SICS, BCVA.

Copyright $(\mathcal{C} 2021$ The Author(s): This is an open-access article distributed under the terms of the Creative Commons Attribution 4.0 International License (CC BY-NC 4.0) which permits unrestricted use, distribution, and reproduction in any medium for non-commercial use provided the original author and source are credited.

\section{INTRODUCTION}

Cataract is one of the major causes of reversible blindness, accounting for about $33 \%$ of cases of visual impairment in the world according to the WHO [1]. Small incision cataract surgery (SICS) is one of the surgical techniques commonly used in developing countries [2-4]. Many patients who present for cataract surgery have eyes complicated by pupils with iris sphincter sclerosis due to aging, synechiae, previous trauma or surgery, diabetes, uveitis, chronic miotic therapy, pseudoexfoliation, or other issues that cause a pupil to dilate poorly [5]. At least $2 \%$ of cases have a pupil that dilates maximally to $5 \mathrm{~mm}$ or less [6]. Performing cataract surgery on an eye with a small pupil remains technically challenging and can cause iris trauma, iris prolapse into a wound, posterior capsular rupture, dropped nuclear fragments and incomplete evacuation of any cortical material [6, 7]. Small pupil in an eye with a hard cataract is prone to more complications.
A number of techniques can be used to mechanically enlarge the pupil during cataract surgery. Most surgical manoeuvres for pupil expansion and prevention of intra operative constriction are less than ideally safe. They can lead to an increased risk of iris sphincter tears, bleeding, iris damage, posterior capsular tears and vitreous loss [7]. Mechanical stretching with two instruments such as microhooks is a relatively simple and effective manoeuvre, but it permanently damages the iris sphincter [8]. The use of iris hooks may also damage the pupillary margin intraoperatively, producing a semimydriatic nonreactive pupil postoperatively [9]. Single-use pupil expansion rings that retract and protect the pupil margin throughout the case are available, but they require special instruments (ring inserter), more time to place, extra care in removal and a significant added expense, ill-afforded by patients in developing nations [10]. 
Cutting the pupillary sphincter is a great method of obtaining a larger pupil during surgery [11]. Multiple tiny sphincterotomies created with smallcalibre vannas scissors can be helpful in a rigid, nondilating pupil and provides excellent post operative results, with a reacting and near-normal sized pupil post-operatively in the majority of patients.

This study was undertaken in economically backward patients who presented to us with hard cataracts and rigid pupils, to study the safety and efficacy of sphincterotomy.

\section{Material ANd Methods}

This study was carried out on 18 patients/20 eyes, who underwent sphincterotomy due to small/nondilating pupil of $<6 \mathrm{~mm}$ with a white/hard cataract during small incision cataract surgery in the Regional Institute of Ophthalmology, PGIMS, Rohtak.

The patients enrolled in the study were $>50$ years of age, with white/ hard cataracts, with $<6 \mathrm{~mm}$ pupils at the time of surgery. Informed consent was taken from all patients. The age and sex distribution was as below:

Table-1

\begin{tabular}{|l|l|l|l|}
\hline \multirow{2}{*}{ Age group of patients } & \multicolumn{2}{|l|}{ Number of eyes } & \multirow{2}{*}{ Percentage } \\
\cline { 2 - 3 } & Male & Female & \\
\hline $50-60$ & & 3 & $15 \%$ \\
\hline $61-70$ & 1 & 3 & $20 \%$ \\
\hline $71-80$ & 4 & 4 & $40 \%$ \\
\hline $81-90$ & 3 & 2 & $25 \%$ \\
\hline
\end{tabular}

The majority of the patients were in the age group of 71-80 years (40\%), which explains the larger nuclear size and advanced cataracts. $60 \%$ of patients were females and $40 \%$ were males.

A complete pre-operative examination was done in all the patients including best corrected visual acuity, slit lamp examination, intraocular pressure measurement, documentation of pupillary size and reaction, A-scan biometry and keratometry. Fundus could be visualized only in two eyes of one patient as there was a mature/dense cataract in the rest of the patients.

Slit Lamp examination revealed corneal degeneration in one of the patients. $20 \%$ patients had a preoperative best corrected visual acuity (BCVA) of $6 / 60-6 / 36$, while $80 \%$ of patients had BCVA of $<6 / 60$.

Table-2

\begin{tabular}{|l|l|l|}
\hline Best corrected visual acuity & Number of eyes & Percentage \\
\hline $6 / 60-6 / 36$ & 4 & $20 \%$ \\
\hline$<6 / 60$ & 16 & $80 \%$ \\
\hline
\end{tabular}

Pre- operative preparation: Tropicamide and phenylepherine eye drops were instilled in all the patients every 20 minutes 2 hours before the surgery, along with topical antibiotic and anti- inflammatory eye drops. Local analgesia required for surgery was achieved by peribulbar block with the infiltration of xylocaine $2 \%$ combined with bupivacaine $0.5 \%$ and hyaluronidase.

\section{Surgical Procedure}

In all these cases of an inadequately dilated pupil of $<6 \mathrm{~mm}$, intraoperative pupillary dilation was attempted by adding adrenaline $0.5 \mathrm{CC}(1 \mathrm{mg}$ for $1 \mathrm{ml}$ ampule) to the $500 \mathrm{ml}$ infusion bottle. A sphincterotomy was done only in the patients in whom the pupil remained $<6 \mathrm{~mm}$ in size. A superior rectus bridle suture was passed. Conjunctival flap was raised and wet-field cautery was done. A frown shaped scleral incision measuring $7 \mathrm{~mm}$ was made, $1.5 \mathrm{~mm}$ from the limbus. Sclerocorneal tunnel was created and entry into anterior chamber was made by a keratome. OVD (ocular viscoelastic devices) was injected into anterior chamber. Before sphincterotomy, the intra-operative pupillary dilation was $4.5 \mathrm{~mm}$ in ten eyes and $5 \mathrm{~mm}$ in rest of the ten eyes. Pupillary sphincterotomy was performed by angled vannas scissors after lifting the pupillary edge by injecting OVDs under it. The first site of sphincterotomy was either 6 or 9'o clock position and if pupillary dilation was less than $6 \mathrm{~mm}$, another sphincterotomy was done 180 degrees away. In one eye, the pupillary size remained less than $6 \mathrm{~mm}$ after 2 sphincterotomies, hence an additional sphincterotomy was done. The anterior chamber was formed with OVDs and anterior capsulotomy was performed with a bent 26 gauge needle by the envelope technique in 6 $(30 \%)$ eyes. In 14 eyes $(70 \%)$ continuous curvilinear capsulorhexis was done. Nucleus was prolapsed in the anterior chamber and removed with an irrigating wire vectis. Residual cortical matter was removed with a simcoe irrigation aspiration cannula. A rigid indigenous posterior chamber intraocular lens, costing less than 4 US dollars, was implanted in $8(40 \%)$ of the eyes, and a foldable IOL in rest of the $12(60 \%)$ eyes. Anterior chamber was washed with balanced salt solution plus and sub conjunctival dexamethasone injection was administered. These surgeries were carried out by the 
same surgeon. Post-operatively, patients were put on topical antibiotics and steroids, which were tapered over 6 weeks.

\section{RESUlTS}

20 eyes of 18 patients with white/hard underwent small incision cataract surgery with posterior chamber IOL implantation with sphincterotomy due to small rigid pupil, $<6 \mathrm{~mm}$. Final outcome was recorded 4 weeks after the surgery.

A pupil size of 6-7 mm was achieved intra operatively after sphincterotomy and surgery was completed with successful implantation of posterior chamber IOL in all the patients. None of the patients had any post-operative complications and none of the eyes showed intra ocular lens dislocation or subluxation.

Table-3

\begin{tabular}{|l|l|l|}
\hline & Number of eyes out of 20 & Percentage \\
\hline Intra operative complications & 0 & $0 \%$ \\
\hline IOL insertion & 20 & $100 \%$ \\
\hline $\begin{array}{l}\text { Pupil-Sluggish reaction } \\
\text { Normal reaction }\end{array}$ & 2 & $10 \%$ \\
\cline { 2 - 3 } $\begin{array}{l}\text { Best corrected visual acuity(postop)- } \\
\text { 6/6-6/9 }\end{array}$ & 18 & $90 \%$ \\
$<6 / 9$ & 18 & 96 \\
\hline
\end{tabular}

Visual acuity of $\geq 6 / 9$ was achieved in 18 (96\%) of eyes. Among the remaining 2 (4\%) eyes, 1 eye had corneal degeneration and 1 had macular degeneration. $90 \%$ of the eyes had a normal pupillary reaction. 2 eyes had an irregular, sluggishly reacting, semi dilated pupil, although the patient had no complaints. These patient had a hard cataract with a rigid pupil of $5 \mathrm{~mm}$ intraoperatively which required more than two sphincterotomies to achieve intraoperative pupil of $7 \mathrm{~mm}$. Some evidence of pigment dispersion on anterior surface of lens was present in this eye with semi dilated pupil.

\section{DisCUSSION}

Cataract continues to be the leading cause of reversible blindness globally. Patients in developing nations like India often present late, with white or hard cataracts. There is an increased risk of complication in eyes with small pupil or non-dilating pupil during cataract surgery $(<6 \mathrm{~mm})[12]$.

A small incision cataract surgery in eyes with a rigid, non dilating pupil of $<6 \mathrm{~mm}$ with white or hard cataract is a challenge and potentially vision threatening and can cause many complications intra and post operatively. Newer methods such as expansion rings and hooks are very expensive and not affordable by patients presenting in government hospitals in the developing countries like India. Several techniques can be used to mechanically enlarge the pupil intraoperartively. Most of them for pupil expansion and prevention of intra operative constriction are less than ideally safe. They can lead to an increased risk of iris sphincter tears, bleeding, iris damage, posterior capsular tears and vitreous loss [7]. Iris hooks may also damage the pupillary margin intraoperatively, producing a semimydriatic nonreactive pupil postoperatively [9]. Single-use pupil expansion rings that retract and protect the pupil margin throughout the case are available, but they require special instruments (ring inserter), more time to place, extra care in removal and a significant added expense, ill-afforded by patients in developing nations [10].

Cutting the pupillary sphincter is a great method of obtaining a larger pupil during surgery [11].

The above study in which we have used a simple, less-time consuming technique of sphincterotomy with vannas scissors shows that this procedure can provide an adequate pupil size in a rigid/non-dilating pupil to perform small incision cataract surgery even in white or hard cataracts, without adding any extra expense or time. No special instrument/equipment is required and the pupil retains a normal size post operatively, while preserving pupillary reaction to light in a large majority of the patients and has excellent post-operative results.

\section{CONCLUSION}

Small pupil is of the most important causes of intraoperative complications during cataract surgery. We suggest that sphincterotomy can be done as a safe and effective procedure, with no special instruments, in non-dilating small pupils of $<6 \mathrm{~mm}$ during SICS. This is especially useful in white and hard cataracts and does not involve any additional cost.

Financial Disclosure: None.

Conflict of Interest: None.

\section{REFERENCES}

1. Causes of blindness and visual impairment. (n.d.). Retrieved March 16, 2017, from http://www.who.int/blindness/causes/en/ 
2. Hennig A, Kumar J, Yorston D, Foster A. Sutureless cataract surgery with nucleus extraction: outcome of a prospective study in Nepal. Br J Ophthalmol. 2003;87:266-270.

3. Hennig A, Kumar J, Singh AK, Ansari A, Singh S, Gurung R, Foster A. World Sight Day and cataract blindness. British journal of ophthalmology. 2002 Jul 1;86(7):830-1.

4. Ruit SPG, Gurung R, Tabin G, Moran D, Brian G. An innovation in developing world cataract surgery. Clin Exp Ophthalmol. 2000;28:274-279.

5. Taklikar AR, Sajjanshetty SV. Intra Operative and Post Operative Complications in SICS Patients with Poorly Dilating Pupil. Ophthalmology and Allied Sciences. 2018;4(1):7-11.

6. Henderson B, Pineda R, Chen S. Essentials of Cataract Surgery. 1st ed. SLACK Incorporated; 2014.

7. Wilczynski M, Wierzchowski T, Synder A, Omulecki W. Results of phacoemulsification with Malyugin Ring in comparison with manual iris stretching with hooks in eyes with narrow pupil [printed online ahead of print October 26, 2012]. Eur J Ophthalmol. 2012. doi: 10.5301/ejo.5000204.
8. Assaf, A., 2013. Cataract Surgery In Small Pupils: Choosing The Appropriate Tool. [online] CRSTEurope. Available at: $<$ https://crstodayeurope.com/articles/2013sep/cataract-surgery-in-small-pupils-online-surveychoosing-the-appropriate-tool/.

9. Vryghem, CJ., 2013. Blind Chopping: Managing Small Pupils Without Pupil-Expanding Devices. [online] CRSTEurope. Available at https://crstodayeurope.com/articles/2013-sep/blindchopping-managing-small-pupils-without-pupilexpanding-devices/.

10. Managing small pupils: A step-wise approach [Internet]. Ophthalmology Times. 2014 [cited 26 March 2017]. Available from: http://ophthalmologytimes.modernmedicine.com/o phthalmologytimes/content/tags/boris-malyuginmd-phd/managing-small-pupils-step-wiseapproach?page $=$ full

11. Colvard D. Achieving excellence in cataract surgery. 1st ed. 2009.

12. Greenberg PB, Tseng VL, Wu WC, Liu J, Jiang L, Chen CK, Scott IU, Friedmann PD. Prevalence and predictors of ocular complications associated with cataract surgery in United States veterans. Ophthalmology. 2011 Mar 1;118(3):507-14. 